\title{
Les recueils de questions-réponses à l'Epoque Moderne : une forme de transmission du savoir scientifique?
}

\author{
Iolanda Ventura \\ CNRS-IRHT, Centre Augustin Thierry/Université d'Orléans ; Labex HASTEC ANR-10-LABX-85, \\ PC 5, COMMENT-R ${ }^{1}$, France
}

\begin{abstract}
Résumé. Mon article traite du genre littéraire des recueils scientifiques de questionsréponses, et en particulier des critères qui permettent une identification et une caractérisation des différents types de textes structurés de cette façon. Ces critères, tels que la structure des unités textuelles formées par les questions et les réponses, la présence et le rôle des sources, la séquence des sujets et l'image du monde de la nature livrée par ces œuvres, permettent aussi de mieux comprendre le rôle et la fonction de chaque texte et type de texte, ainsi que du genre littéraire en général, dans le mouvement de transmission du savoir au Moyen Age, à la Renaissance et à l'Epoque moderne. A l'aide de ces critères, je livre une analyse de deux recueils écrits au XVI et au XVII ${ }^{\mathrm{e}}$ siècle, les Problemas de Francisco Lopez de Villalobos et les Pensieri diversi d'Alessandro Tassoni.
\end{abstract}

\begin{abstract}
Collections of questions-and-answers in the Modern Area: a form of transmission of scientific knowledge? My article deals with the literary genre of the scientific collections written in questions-and-answers form. More particularly, I will describe the criteria I use in order to identify and catalog the various texts structured in this specific form, and to understand the way in which they contribute to the transmission of scientific knowledge. That criteria can be identified in the structure shown by each textual unity consisting in question + answer, in the mention of the sources and the function they fulfill in the text, in the sequence of topics the compilers deal with and the image of the natural world they deliver. With the help of such criteria, I will examine two collections written during the $16^{\text {th }}$ and the $17^{\text {th }}$ century, Francisco Lopez de Villalobos' Problemas and Alessandro Tassoni's Pensieri diversi.
\end{abstract}

\section{Introduction}

Pendant les dernières décennies, les textes encyclopédiques, la culture encyclopédique et l'encyclopédisme ont attiré l'attention d'un nombre croissant de chercheurs ${ }^{2}$. En particulier, le nouvel intérêt montré par la recherche pour les textes encyclopédiques a contribué à les faire changer de statut,

\footnotetext{
${ }^{1}$ Ce texte reprend le contenu de deux séminaires données en Septembre 2012 et en Septembre 2014. Je remercie les participants à ces séminaires, et en particulier Mme Jeanne Peiffer, M. Bernard Vitrac, et Mme Francesca Aceto. Un merci particulier va à mes relecteurs, Mme Giovanna Cifoletti et M. Stéphane Lamassé, et à M. Alain Bernard, sans lequel ce texte n'aurait jamais été écrit.

${ }^{2}$ Sur la littérature encyclopédique du Moyen Age, de la Renaissance et de l'époque moderne, voir les volumes collectifs (Picone 1994, Meier 2002, De Callataÿ et Van den Abeele 2009, Zucker 2014).
}

This is an Open Access article distributed under the terms of the Creative Commons Attribution License 4.0, which permits unrestricted use, distribution, and reproduction in any medium, provided the original work is properly cited. 
en les transformant de mélanges issus d'un simple travail de « copier-coller » complètement dépourvu d'esprit critique et d'originalité en moyens privilégiés de collection, de transmission, de réception et d'assimilation du savoir aux différentes époques. Une des méthodologies utilisées par les chercheurs -et pour cette étude, la plus intéressante- consistait à mettre en relation les textes encyclopédiques avec d'autres formes de collection, d'organisation et de transmission du savoir tels que les summae ou les compendia philosophiques et scientifiques d'un côté, et les recueils consacrés aux sciences théoriques (e.g., astronomie ou mathématique) ou aux « savoirs pratiques » (alchimie, pharmacie, parfumerie et cosmétique) de l'autre. En montrant les points communs et les différences entre ces typologies de texte et de transmission du savoir, la recherche - et en particulier les études concernant la Renaissance et l'Epoque Moderne - a pu abstraire des textes encyclopédiques des notions plus théoriques qu'on a pu mettre à la base d'une réflexion plus ample, comme par exemple celle de «culture encyclopédique » et d' « encyclopédisme ». Ces notions ont aussi acquis des significations propres : la première traduit, grosso modo, la tentative de structurer, à travers les sources utilisées par les compilateurs et les disciplines scientifiques qu'ils décrivent, l'arrière-plan culturel d'une époque et de le synthétiser dans une compilation. La seconde traduit, de son coté, l'ambition plus large et profonde de maîtriser un savoir universel et total, englobant tous les éléments du monde, tous les savoirs qui l'examinent, et tous les livres qui en parlent.

En dépit des nombreuses recherches, les notions de texte encyclopédique, de culture encyclopédique, et d'encyclopédisme ne sont toutefois ni bien établies ni toujours aperçues de la même manière. Au contraire, presque chaque chercheur travaillant sur les encyclopédies de l'Antiquité, du Moyen Age, de la Renaissance et de l'Epoque Moderne a mis à la base de ses travaux une notion d'encyclopédie et de background culturel et intellectuel susceptible d'être défini comme « encyclopédique », en rendant parfois le dialogue à l'intérieur de la recherche scientifique très complexe, voir impossible ${ }^{3}$.

La situation se complique ultérieurement quand on cherche à voir une attitude ou une ambition encyclopédique en termes d'intention de recueillir, organiser, transmettre et rendre accessible un savoir « universel ». En premier lieu, c'est la notion même d'universalité qui pose problème, car elle peut, en même temps, indiquer un savoir global regroupant toutes les disciplines, mais aussi un savoir extrêmement riche et profond concernant une seule discipline, et la reflétant dans toutes ses formes et contenus. En outre, il est parfois peu évident de retrouver cette notion (ou ambition) d'universalité dans des textes qui ne sont pas des vraies « encyclopédies » (car ils n'ont ni de caractère universel dans leur contenu, ni un titre qui renvoie à une universalité du savoir), mais qui relèvent d'un esprit encyclopédique comme les recueils de secrets, les dialogues savants traitant du monde de la nature, les dictionnaires et les recueils de «lieux communs ». Cet élargissement de sens pose une question qui est, et reste, la suivante : le fait d'avoir traité d'un ensemble de disciplines et de notions variées, d'avoir eu l'ambition de livrer une multitude de notions, de reproduire et de mettre à disposition un savoir plus vaste, sans pourtant avoir le but de reproduire dans un livre la totalité du monde de la nature et/ou du système des sciences, justifie-t-elle l'insertion de ces œuvres à l'intérieur d'une culture encyclopédique, l'interprétation de ces textes comme « recueils encyclopédiques », la définition de ces œuvres comme moments de l'histoire de l'encyclopédisme?

Cet article ne peut ni ne veut livrer une réponse générale, mais cherche, plus modestement, à aborder ces questions de catalogage, de définition et d'interprétation en traitant d'un genre littéraire bien précis, les recueils de questions-réponses ayant pour objet l'histoire naturelle à la Renaissance et à l'Epoque Moderne. Ces textes font partie de plein droit des systèmes de transmission et de diffusion du savoir, et bien au delà du monde occidental. Comment peut-on les caractériser et les distinguer à l'intérieur de la littérature scientifique et encyclopédique?

\footnotetext{
3 Voir à ce propos le volume collectif (König et Woolf 2013), ici p. 4-5 («Introduction»). Voir aussi la contribution de C. Macé dans ce volume.
} 


\section{Les recueils des questions-réponses dans la recherche}

L'intérêt pour ce genre de texte a déjà caractérisé le travail de plusieurs générations de chercheurs. Après l'étude pionnière de Brian Lawn, qui avait livré, en 1963, une histoire des recueils de questions-réponses et des dialogues scientifiques et philosophiques au Moyen Age et à la Renaissance et avait contribué à faire connaître le «système question-réponse » comme stratégie de transmission du savoir ${ }^{4}$, ce genre littéraire a déjà fait l'objet, il y a quelques années, des recherches d'Ann Blair, qui a essayé dans deux articles publiés en 1999 de livrer une définition de ce type de texte ${ }^{5}$. Celle proposée par la chercheuse n'arrive pas à couvrir l'éventail des textes qu' on peut définir comme « recueils de problèmes ». En fait, le centre d'intérêt de l'analyse d'A. Blair s'identifie avec les recueils intitulés Problemata et appartenant, au moins dans les intentions, à la tradition des Problemata pseudo-aristotéliciens. Elle implique donc que, dans sa perspective, les recueils de «problèmes » s'identifient plus ou moins totalement avec la perspective de l'œuvre attribuée à Aristote, à savoir l'identification de la démarche logique qui règne sur un phénomène naturel et permet de l'expliquer, et surtout de la cause qui est à l'origine du même phénomène ${ }^{6}$.

Il y a quelques années, j'ai repris le dossier dans quelques études en adoptant une approche sensiblement différente de la chercheuse américaine. Le but de mes recherches n'était pas d'identifier un genre de texte unique et une seule tradition textuelle de recueils de questions-réponses, mais de présenter un éventail plus large d'œuvres, de formes, de structures de textes, de niveaux intellectuels et culturels ${ }^{7}$. Bref, il s'agissait pour moi de décliner la typologie textuelle du recueil de questions-réponses dans ses multiples formes et manifestations entre Moyen Age et Renaissance, et de tenter une définition de ce genre littéraire qui pourrait mieux l'identifier et le distinguer par rapport à celui des dialogues fictifs ${ }^{8}$ ou des miscellanées encyclopédiques ${ }^{9}$. Ce qui m'intéressait plus particulièrement était l'utilisation et la transformation de l'arrière-plan intellectuel et culturel montrées par les compilateurs des recueils de questions-réponses. La question n'était pas limitée à l'identification des textes de référence : le plus important était de comprendre la manière dont ils les utilisaient et la façon de véhiculer à leurs lecteurs non seulement la réponse à une question et l'explication ou la cause d'un phénomène naturel précis, mais tout un bagage de connaissances variées liées de manière directe ou indirecte avec le phénomène en question. En d'autres termes, il s'agissait de comprendre leur valeur comme forme de transmission des connaissances et comme typologie de texte savant à lire avec plusieurs buts : en premier lieu, pour comprendre la cause d'un phénomène naturel; ensuite, pour acquérir des connaissances plus vastes dans le domaine du savoir concerné par le phénomène (météorologie pour le ciel sublunaire, les vents, les phénomènes atmosphériques, zoologie pour les animaux, etc.) ; enfin, pour apprendre la logique interne au monde de la nature (pour ainsi dire, de percer ses causes internes). Pour atteindre cet objectif, il n'était pas suffisant de s'appuyer sur quelques textes-phares en cherchant de dériver, à partir d'une petite sélection, les éléments représentatifs qui permettaient de tracer l'identité du genre littéraire dans son évolution historique ; on devait, en premier lieu, mettre ensemble un catalogue des textes susceptibles de faire partie d'une recherche plus vaste, et structurer ce même catalogue sur la base de critères précis,

\footnotetext{
4 Voir Lawn 1963.

5 Voir Blair 1999a.

${ }^{6}$ Cf. Blair 1999b, ici p. 173: "Although the tightly paradoxical formulation of the Aristotelian problema loosened over time, the genre of natural philosophical and medical problemata involved exclusively causal questions (in "why ?" and synonyms), applied to commonly known particular phenomena. The genre was built around an Aristotelian conception of philosophical knowledge as causal knowledge, applied to the explanation of ordinary rather than specialist or abstract questions".

7 Cf. Ventura 2004, 2007, 2009, 2015.

8 Sur le genre littéraire des dialogues, voir, pour l'Antiquité tardive et le Moyen Age, la synthèse dans Cardelle de Hartmann 2007, et pour la Renaissance, celle offerte par le volume collectif (Hempfer 2002), et par la monographie de Traninger 2012. Voir aussi le volume collectif (Bergdolt et Pfister, 2011).

${ }^{9}$ Sur le genre littéraire des miscellanées encyclopédiques, et en particulier sur les Quaestiones mensales de Plutarque, voir le volume collectif Klotz et Oikonomopoulou 2011.
} 
capables de situer chaque texte dans une catégorie précise. La question primaire était, et est encore, représentée par l'identification de ces critères.

\section{Cataloguer les recueils de questions-réponses : critères et questions}

Avec le but d'écrire une histoire du genre littéraire des recueils de questions-réponses et de tracer une typologie de ces textes, j'ai commencé à dresser, au fil des années, un petit catalogue des recueils de questions-réponses écrits entre le Moyen Age et l'Epoque Moderne, en me basant d'abord sur l'aperçu de l'évolution de ce genre littéraire donné par B. Lawn dans son étude The Salernitan Questions ${ }^{10}$. La littérature est vaste, mais ce n'est pas son ampleur qui pose principalement problème. La vraie question à la base de la constitution d'un tel catalogue concerne l'identification des critères les plus efficaces à utiliser. Ces critères servent, en même temps, à identifier les recueils de questions-réponses et à les distinguer des autres genres de transmission de savoir autres que les traités scientifiques et les textes encyclopédiques comme les dialogues fictifs, les recueils de lieux communs, les romans érudits ${ }^{11}$. Ils visent en outre à leur trouver une place dans le processus de diffusion de la culture scientifique parmi les savants et les érudits qui, tout en n'étant pas des spécialistes des différentes disciplines, montraient un intérêt bien réel et des moyens intellectuels aptes à comprendre les informations issues de disciplines spécifiques ${ }^{12}$. Enfin, ces critères ne peuvent pas être seulement extérieurs : on ne peut pas, par exemple, prendre en considération seulement les œuvres intitulées «Problemata» ou «Quaestiones », car ce titre est trompeur. Il peut se retrouver, en fait, non seulement à la tête de recueils de questions-réponses, mais aussi d'œuvres académiques comme des disputationes, ou des manuels spécialisés comme les recueils de médecine structurés sur le modèle du Conciliator de Pierre d'Abano (e.g., le Conciliator enucleatus de Gregor Horst ${ }^{13}$ ou lesProblemata miscellanea de Iohannes Jacobi ${ }^{14}$ ). Il fallait donc trouver d'abord des critères internes aux textes, et relatifs à leur structure, au choix et au traitement de la matière choisis par le compilateur, et à l'image du monde et du savoir livré par la compilation.

\section{1 les critères de division et structuration du texte}

Dans cet esprit, le premier critère de catalogage est, évidemment, la division du texte en questions et réponses. Bien que primaire, ce critère n'est pas toutefois à accepter sans apporter des précisions. En premier lieu, il faut examiner la question posée et sa nature, pour voir si elle part d'un intérêt ponctuel pour un objet/phénomène naturel, pour ses causes et pour ses manifestations plus ou moins exceptionnelles, ou si elle reflète un débat plus large (comme par exemple la génération spontanée), examiné par l'auteur en partant d'un exemple spécifique. En d'autres termes, il faut essayer de comprendre, dans l'œuvre, si les questions posées montrent une attention plus concrète pour un objet, ou si elles reprennent consciemment une discussion scolastique plus profonde et cherchent soit à la rendre plus accessible à leurs lecteurs en la liant à un cas concret, soit à la rappeler dans toutes ses nuances et ses complications. Il faut aussi chercher à comprendre dans quel rapport se trouvent la question et sa réponse. Dans les recueils de questions-réponses, on peut ainsi trouver des exemples de questions très brèves et ponctuelles, suivies par des réponses tout autant brèves et ponctuelles, mais aussi des cas qui montrent une relation plus complexe entre questions et réponses. Dans ce dernier cas, la première

\footnotetext{
${ }^{10}$ Lawn 1963.

${ }^{11} \mathrm{Cf}$. l'Introduction à ce volume, Sect. 2.2.2

12 À ce propos, il faut remarquer que la recherche en matière d'histoire des encyclopédies n'a pas encore essayé de donner une définition exacte de la notion de « lecteur non-spécialisé », qui n'indique pas forcément un lecteur «incompétent », ni de la présenter dans ses différentes nuances. Je remercie Jeanne Peiffer (CAK, CNRS, Paris) d'avoir attiré mon attention sur la nature complexe de cette notion.

13 Horst 1621.

14 Jacobi 1662.
} 
devient un point de départ, on pourrait dire un prétexte pour le plein développement de la seconde, dont la dimension et la profondeur, le contenu et la bibliothèque des sources dépassent largement la «banalité » de l'objet/phénomène naturel présenté comme problématique. Evidemment, l'éventail des possibilités entre ces deux extrêmes peut être, à son tour, large.

\subsection{Les sources, leur choix et leur traitement}

Un deuxième critère de catalogage et d'interprétation des recueils prend en considération les sources. Dans le cas des recueils de questions-réponses, il ne s'agit pas seulement d'identifier les sources utilisées par les compilateurs et de définir les stratégies de leur traitement, mais aussi d'établir le rôle joué par la ou les sources dans l'émergence de la question. A titre d'exemple, on peut présenter la question de manière différente : dans le cas des recueils de questions-réponses, le compilateur peut utiliser sa ou ses sources non seulement pour livrer une réponse certaine et pourvue d'autorité, garantie par le nom de l'auteur et de l'œuvre cités et repris, mais aussi pour dériver d'un passage de la source même une possible question artificielle, en donnant le passage comme réponse ${ }^{15}$. Par conséquent, il n'est pas seulement important de comprendre si, dans le cas de la zoologie, il préfère utiliser les Historiae animalium d'Aristote, la Naturalis historia de Pline l'Ancien, ou le De animalibus d'Albert le Grand (ou les trois textes ensemble), mais de voir de quelle manière il manipule la source pour transformer une affirmation en un objet de doute et, donc, de question.

\subsection{La structure de la compilation et l' « image du monde »}

Le troisième critère prend comme point de départ la structure du texte, la séquence des questionsréponses, le choix des sujets et leur encadrement. Ce troisième critère se concentre sur des aspects du texte qui se révèlent très importants pour comprendre la volonté, de la part du compilateur, de rédiger une œuvre cohérente qui utilisent la forme question-réponse ou de mettre ensemble une séquence de questions plus ou moins pertinentes au regard de l'intention globale. Dans les deux cas, ce n'est pas seulement la structure qui compte, mais aussi le but et l'intention de l'auteur qu'elle cache : dans le cas d'un recueil « éclaté », sa raison d'être pourrait se retrouver dans la varietas et dans le plaisir intellectuel qu'elle procure plutôt que dans l'harmonie et la cohérence interne d'un système et dans l'enseignement qu'il offre ${ }^{16}$. Le contraire vaut, évidemment, pour une structure compacte, où la séquence des questions est encadrée dans un système logiquement cohérent, où les sujets et les questions se succèdent en suivant un plan bien précis. Par exemple, une séquence de questions vue en tant qu'ensemble et dans le passage d'un sujet à l'autre montre l'image que le compilateur veut fournir de l'univers du ciel à la terre et de son décor, ou des animaux classifiés en quadrupèdes etc. ${ }^{17}$

Plus précisément, il faut voir si l'ensemble des questions-réponses ou, au moins, une partie entre elles, forme un noyau cohérent, montre une véritable séquence logique des sujets approchés ; à savoir, si par exemple une série de problèmes traitant du monde animal à l'intérieur d'un recueil plus varié, ou une œuvre strictement divisée en livres ou en sections, chacun(e) desquels consacré à un sujet spécifique. Donc, la question de la structure du texte est à aborder à un double niveau, à savoir en prenant en considération l'entièreté du texte autant que les sections dont il est constitué ${ }^{18}$. En outre,

\footnotetext{
15 Cf. sur ce sujet Ventura 2009 et 2015. Les deux études prennent comme point de départ la Catena aurea entium, une gigantesque encyclopédie en 10 livres structurée sous forme de questions-réponses écrite par le dominicain Henry d'Herford pendant la seconde moitié du XIV ${ }^{\mathrm{e}}$ siècle, et explorent la technique de constitution de cette compilation, et la relation entre questions et réponses.

${ }^{16}$ Cf. la contribution de G. C. Cifoletti dans ce volume.

17 Voir la séquence de questions-réponses incluses dans le Responsorium curiosorum attribué à Conrad d'Halberstadt, publié à Lübeck en 1476 (version digitale accessible au site http://digital.staatsbibliothek-berlin.de/werkansicht $/$ ?PPN $=$ PPN739818120) .

$18 \mathrm{Cf}$. l'Introduction à ce volume, Sect. 2.1.5.3.
} 
la présence, ou l'absence, d'une structure et d'une cohérence interne dans le recueil, doit être prise en considération, car elle offre des éléments ultérieurs d'évaluation de la nature, du Sitz im Leben, à savoir, de son rôle et de sa place dans la culture du temps, des modalités de lecture et d'utilisation, et des buts de la compilation. Par exemple, on peut se demander pour quel type de lecture soit conçu un recueil apparemment dépourvu de structure, et basé sur le principe de la varietas et de la polymathia plutôt que sur celui du traitement ordonné des sujets ou sur une hiérarchie des éléments naturels et/ou des disciplines. Pour mieux dire : est-il conçu pour la consultation ponctuelle, pour la lecture in extenso, ou bien tout simplement pour amuser le lecteur avec son choix des sujets et le contenu de ses réponses (un emploi strictement pédagogique au sein d'une institution de formation me semble, au moins pour les textes écrits à la Renaissance et à l'Epoque moderne, peu probable) ?

Pour évaluer la nature, la destination, les modalités de lecture des recueils de questions-réponses, il est pourtant nécessaire de prendre en considération plusieurs aspects : à coté, évidemment, du contenu, ce sont les éléments paratextuels qui l'accompagnent qui méritent une attention spécifique. En particulier, il faut considérer les préfaces écrites par l'auteur et/ou l'imprimeur, une partie du texte souvent utilisée par les auteurs et/ou les imprimeurs pour livrer une « fiche d'identité » du recueil et tracer le portrait de son lecteur idéal. Autres éléments distinctifs, quand ils sont présents, sont les index qui, surtout dans le cas d'œuvres apparemment incohérentes, guident le lecteur vers le repérage immédiat des questions susceptibles de l'intéresser, et/ou offrent des regroupements et des systèmes de navigation du texte alternatifs (par exemple, dans le cas où ils réunissent dans la même séquence des questions consacrées au même sujets, mais éparpillées à l'intérieur du texte).

$* * *$

Les critères sélectionnés pour un premier catalogage sont en rapport avec la représentation, la structuration, la mise en question, et la transmission du savoir dans les œuvres. Ces critères, qui sont aussi à la base de l'analyse des textes encyclopédiques, doivent être appliqués dans le cas des recueils de questions-réponses en tenant compte des conditions mentionnées dans les paragraphes précédents, pour permettre à ce genre de texte et aux œuvres qui le caractérisent de trouver sa place et d'émerger à l'intérieur de la littérature encyclopédique médiévale, de la Renaissance et de l'Epoque Moderne, ainsi que des formes de texte qui ont l'ambition de transmettre le savoir. Pour mieux exemplifier les critères et les difficultés d'un catalogage des recueils de questions-réponses écrits pendant la Renaissance et l'Epoque moderne, je discuterai deux exemples dans les paragraphes suivants (Sects. 4 et 5). Ces deux textes ont en effet en commun le fait d'être structurés dans cette forme, mais d'être aussi profondément différents pour ce qui concerne la nature et la forme des réponses et leur rapport avec les questions de départ, leur arrière-plan intellectuel, leur présentation, leur destination, et leurs modalités de lecture et d'utilisation.

\section{Un recueil « ludique » de question-réponses : les Problemas de Francisco Lopez de Villalobos}

Le premier texte considéré dans notre étude sont les Problemas du médecin juif espagnol Francisco Lopez de Villalobos (c. 1473 - c. 1549), un auteur connu surtout pour son poème médical concernant la syphilis, un des premiers témoignages littéraires de cette maladie écrits en Espagne ${ }^{19}$. Les Problemas

\footnotetext{
${ }^{19}$ Lopez de Villalobos 1543 (publié avec les Dos dialogos de medicina, il Tratado de las tres grandes, une Cancion, et la Comedia de l'Ampytrion; la pagination du texte est très aléatoire et dépourvue de cohérence interne entre une numération en folios et en page). Le volume est entièrement numérisé sur www.books . google.com. Sur la biographie de Lopez de Villalobos, voir Arrizabalaga 2002.
} 
sont, en revanche, peu fréquentés par les historiens de la littérature, à l'exception des spécialistes de littérature et de la culture scientifique en terre d'Espagne à la Renaissance. Le texte, publié en 1543, se compose de 41 questions et réponses structurées en 2 livres, dont le premier, consacré au monde de la nature, comprend seulement 6 questions, tandis que le second, conçu autour de l'homme et des animaux, et en particulier de leur vie et de leurs coutumes, en contient $34^{20}$. Les Problemas sont complétés dans l'édition imprimée par 2 dialogues autour de fièvres interpolatae (c'est-à-dire, avec des rechutes à des intervalles de temps fixes, pour le premier), et de la chaleur naturelle de l'homme (pour le second), et par la reproduction d'une conversation entre Villalobos et un dignitaire du royaume de Castille ${ }^{21}$. La structure des questions-réponses est apparemment assez simple, mais, à bien voir, assez particulière : chaque sujet est traité sur la base, on peut facilement imaginer, d'une question spécifique, suivie par sa réponse. La forme et la définition de « question » et « réponse » présente déjà des caractéristiques particulières. Dans ce recueil, la question est présentée sous forme d'un petit poème rimé défini comme « metro » (c'est-à-dire, comme « partie poétique »), tandis que la réponse est conçue et présentée comme une «glose » (« glossa ») au dit poème. L'ampleur de cette glose dépasse toutefois largement la question en soi, et vise à transmettre des connaissances plus génériques. Pour ne donner qu'un exemple : la première question du premier livre, celle qui ouvre la collection, concerne les mouvements du soleil, la durée du jour solaire, qui est plus court de celui d'autres corps célestes, et la théorie des épicycles. Elle est synthétisée dans les vers suivants : "Porque el Sol desde su sphera/haze un dia naturàl/menor que otro ques su ygual/siendo toda una carrera?/Y porque sus compañeros/Mercurio $y$ Venus conel/delanteros o çagueros/tampoco se apartan del? » ${ }^{22}$. La réponse ou « glossa » prend plusieurs pages, mais, plutôt que de donner une solution à la question spécifique, elle se borne à offrir surtout, et de manière très simple et didactique, une description et une explication complète des trois mouvements du soleil. C'est seulement à la fin qu'elle aborde la question des différences entre le jour solaire et celui de Mercure et de Vénus et la description des épicycles.

Donc, a priori, on ne peut pas parler ni d'une relation étroite entre question et réponse, ni d'un rapport complexe entre une question apparemment «banale » (ce qui n'est pas toujours le cas des Problemas qui contiennent des questions très profondes et complexes, de "vrais problèmes scientifiques ${ }^{23}$ ) et une réponse fortement chargée d'érudition, ni enfin d'un « problème » qui reproduit un débat scientifique d'envergure. Dans le cas de Lopez de Villalobos, la question et la réponse trouvent leur valeur et leur importance dans sa capacité d'intéresser un public plus large, de lui transmettre, en même temps, un savoir de haut niveau et de l'amusement, et enfin de le faire avancer, à travers un processus didactique bien planifié et structuré, vers des connaissances plus générales, y compris concernant des disciplines complexes comme l'astronomie ou la théorie des éléments. Cette valeur et cette fonction sont également mises en évidence par la structure même de la réponse, dans laquelle, à ma connaissance, aucun renvoi à une source écrite n'est présent. Cela n'implique pas que le compilateur n'avait pas accès à une bibliothèque plus ou moins vaste, mais seulement un processus de filtrage et de mise en valeur de l'information au détriment des textes de référence. Enfin, la réponse est structurée de manière strictement déductive, marquée par l'utilisation de stratégies linguistiques capables de lier fortement un passage à l'autre ${ }^{24}$.

\footnotetext{
${ }^{20}$ Les deux dernières questions, concernant respectivement la flatterie vis-à-vis des rois et ses conséquences négatives, et les menteurs, sont dédiés au roi Felipe II.

${ }^{21}$ Une note marginale écrite au f. 132v de l'édition consultée pendant la préparation de cette étude identifie le dignitaire avec le duc d'Alba.

22 Lopez de Villalobos 1543, f. 3v.

23 Voir à ce propos les questions 4 et 5 du livre I, concernant la théorie des éléments, ibid., f. 16r-23v.

24 Voir, par exemple, la description du mouvement du soleil d'Orient à Occident (ibid., f. 4 [recte: 3v]-5r): « .. El primero [movimiento] es el que vemos que haze ca da dia de Oriente y Poniente. Y este se cumple en veynte y quatro horas yguales, poco mas. Conviene a saber desde que parte de Oriente hasta que rodeando todo el mundo por arriba y por abaxo buelvue a salir otra vez. Yeste se llama dia natural que comprehende dia y noche. $Y$ desta manera es tam grande el dia de invierno como el de estio,
} 
La particularité de la structure de chaque « unité question-réponse », ainsi que la forme, la nature et la fonction de la question et de la réponse méritent aussi d'être soulignées, car elles nous disent beaucoup sur la nature de l'œuvre. En premier lieu, elle montre que, pour notre auteur, c'est la question qui joue le rôle vedette, qui doit attirer l'attention du lecteur, tandis que la réponse ne devient qu'un accompagnement du problème énoncé dans le « metro » (à savoir, dans la partie versifiée), ainsi qu'un point de départ pour transmettre un savoir et un enseignement plus généraux. La forme et la structure de la réponse, dépourvue de toute référence à des sources et à des autorités, et caractérisée par un ton intentionnellement didactique et rigidement déductif, ainsi que la nature « littéraire » de la question, nous font, de l'autre coté, comprendre que Lopez de Villalobos utilise le modèle question-réponse pour offrir à ses lecteurs une œuvre qui mêle l'esprit pédagogique à une intention « ludique », c'està-dire à une intention de mettre en place une forme de transmission du savoir où le «plaisir de lire et d'apprendre » joue un rôle majeur. L'absence de renvois aux sources est également liée à cette dimension ludico-didactique : elle ne doit être lue ni comme un signe d'ignorance du compilateur (un médecin cultivé) ni de ses lecteurs (qui, au moins en partie, avaient accès à d'autres sources), ni comme une intention de « populariser » la culture scientifique qu'il transmet qui, vu le niveau des questions, ne peut pas être définie comme «populaire ». Plus probablement, il ne cite pas de sources pour la simple raison qu'il n'a pas besoin de soutenir son savoir à l'aide d'une autorité. La nature linéaire de son raisonnement est déjà suffisante à transmettre les informations sans provoquer ni de doutes, ni d'autres questions. En outre, il est plus probable que ses lecteurs, qui lisaient le volume de Lopez pour satisfaire leur curiosité sans alourdir leur travail, n'avaient pas besoin de renvois à d'autres sources, mais seulement de lire et d'apprendre de manière peu compliquée.

Un dernier mot doit être dit sur la structure du recueil et la séquence des questions. Pour mieux les comprendre, on peut parcourir à titre d'exemple la structure du livre I. Si la première question concerne les mouvements du soleil, la deuxième a pour sujet la lumière de la lune, la troisième traite des quatre éléments (terre, air, feu, eau) qui forment le cosmos, leur opposition en tant que tels, leur mélange à l'intérieur des objets, et la prééminence parmi eux du feu. Quant à la quatrième -une des questions plus longues et complexes-, elle s'intéresse à la fonction du feu céleste, la cinquième aux convergences et les similarités entre les éléments. Enfin, la sixième aborde le problème de l'altitude du Paradis terrestre et la possibilité qu'Adam, en raison de cette altitude, aurait pu toucher la lune avec les mains simplement en montant sur un arbre (sic!). Apparemment, ces six questions ne livrent pas des connaissances complètes, mais isolent et traitent des problèmes spécifiques. En conséquence, on pourrait parler des informations livrées par Lopez de Villalobos comme d'un savoir partiel, ponctuel, et sélectionné sur la base des difficultés qu'il présente. Si cette conclusion ne peut pas être niée si on prend en considération exclusivement l'argument des questions, il est vrai aussi que, du point de vue des réponses, l'ampleur et la généralité de la «glose », qui aborde le sujet à son niveau le plus élémentaire, permet au compilateur, avant de répondre à la question spécifique, d'offrir à ses lecteurs un petit traité général concernant l'objet de la réponse et la discipline ou le domaine du savoir concerné. On a déjà vu ce phénomène pour la première question discutée plus haut. Donnons un nouvel exemple : la longue réponse à la troisième question débute avec un petit rappel des connaissances de base à propos de la théorie des éléments, et seulement après aborde les différents aspects problématiques (opposition et mélange des éléments, prééminence du feu) contenu dans le «metro ${ }^{25}$. Par conséquent, on peut affirmer que, bien que la séquence des questions semble présenter une série de questions isolées et

\footnotetext{
porque lo que se acorta del dia se alarga en la noche. Este movimiente se llama diurno, porque se haze cada dia: y llamase rapto, porque el cielo, o sphera donde esta el Sol es arrebata do y traydo por fuerça des primer cielo mobile, que es tan grande y tan potentissimo en su curso, que como el se mueve de Oriente a Poniente, y da una buelta entera en un dia natural, trae consigo arrebatados y forçados a todos los cielos que esta de l'axe des y hazeles dar una buelta cada dia, y hazer el movimiento diurno (como dicho es) en veynte y quatro horas... ».

25 Ibid., f. 10v-16r.
} 
ponctuelles, sans véritables liens internes, celle des réponses montre une volonté de livrer aux lecteurs, à travers l'association de quelques questions, une connaissance plus vaste, bien que non complètement exhaustive. Une telle exhaustivité n'était d'ailleurs probablement pas dans les intentions du compilateur, qui voulait livrer une œuvre amusante et édifiante, plutôt qu'un traité savant ${ }^{26}$.

L'exemple des Problemas de Villalobos montre comment les trois critères de catalogage choisis (structure des unités textuelles, présence et utilisation des sources, forme et division du texte et choix/séquence des sujets) nous permettent d'identifier, au delà des particularités de la collection, une typologie de texte et d'utilisation de la forme « question-réponse » avec une identité précise et des buts spécifiques. Il montre aussi que ces critères ne peuvent pas être considérés comme des valeurs absolues, mais donnent lieu à toute une série de « sous-critères ». Ils demandent donc à être utilisés de manière flexible.

\section{Un recueil « érudit » de questions-réponses : les Pensieri diversi d'Alessandro Tassoni}

Notre deuxième et dernier exemple se situe apparemment à un niveau diamétralement opposé à celui des Problemas de Lopez de Villalobos, et constitue un type de recueil de questions-réponses complètement différent. En réalité, seulement une analyse plus précise pourra confirmer si cette impression est légitime.

Le recueil des Pensieri diversi fut publié pour la première fois en $1608^{27}$; il fut ensuite revu plusieurs fois et imprimé sous forme différente en 1612, 1613, et enfin en 1620, dans la version en 10 livres que nous connaissons aujourd'hui et que nous traitons dans ce paragraphe ${ }^{28}$. Leur auteur, Alessandro Tassoni (1565-1635), est une des personnalités clés de la littérature italienne de l'époque baroque, connu en particulier à cause de la rédaction d'un poème-parodie des chansons de geste à grand succès, $L a$ secchia rapita (Le seau enlevé). Les Pensieri diversi, en dépit de leur succès au XVII ${ }^{\mathrm{e}}$ siècle, n'ont pas véritablement attiré l'attention des chercheurs. Selon toute probabilité, leur nature d'œuvre érudite caractérisée par l' " information overload» typique de l'âge du baroque italien a contribué à son oubli et à son exclusion des histoires de la littérature italienne. Bien que parfois difficile à lire et à approcher, elle mérite notre attention dans cette étude.

Les Pensieri diversi, contrairement aux Problemas de Lopez de Villalobos ont, au moins dans leur version définitive, une structure comparable à celle d'une véritable imago mundi, d'une encyclopédie universelle englobant l'entièreté du monde de la nature. Ses 10 livres traitent, respectivement, de la conception de la matière et de la forme (livre I), du firmament et des étoiles (livre II), du soleil et de la lune (livre III), de l'air, de l'eau et de la terre (c'est à dire, des trois autres éléments qui composent l'univers ; livre IV), des propriétés et des accidentia des objets naturels et des êtres vivants (livre V), de la vie humaine et de ses caractéristiques (livre VI), de la littérature (livre VII), de la vie sociale et politique de l'homme (livre VIII), de l'histoire et de la poésie (livre IX), des biographies des hommes illustres (livre X). Tous les livres sont structurés sous forme de questions-réponses, sauf le dernier qui, selon toute probabilité, ne pouvait pas être structuré de cette façon, car les biographies ne se laissent pas synthétiser facilement en une série de questions-réponses, mais seulement en forme de petits chapitres.

\footnotetext{
26 Voir à ce propos le lieu commun utilisé par l'imprimeur dans l'épitre de dédicace à Diego Nuñez Perez, qui parle des Problemas comme d'un « apazible jardin, hallara diversidad de floridos dichos : unos que le sirvan de solo pasatiempo, y otros, que sirvan de medicina para el cuerpo, y otros de consejos para el alma », à savoir d'un beau jardin de dits, dans lequel on trouve plusieurs fleurs, certains qui ne sont qu'un divertissement, certains qui offrent un médicament pour le corps, et certains enfin qui donnent des conseils relatifs à la vie spirituelle. Je me demande toutefois si dans cette image on ne doit pas voir, comme j'indique dans cet étude, seulement une référence à la nature amusante et savante des questions en général, ou bien aussi une légère dépréciation de celles comprises dans le livre I.

27 Pour cette étude, j'utilise l'édition Tassoni 1645, accessible sur le site www . books. google.com.

28 Sur la biographie et l'oeuvre de Tassoni, voir Fassò 1937. Sur cet auteur, voir aussi le volume collectif (Jannaco 1966).
} 
Par conséquent, et contrairement au cas des Problemas, nous avons à faire avec une œuvre à la forme plus solide et compacte, structurée sur deux piliers ou axes principaux, à savoir l'univers composé des quatre éléments (objet des 5 premiers livres, bien que le cinquième doit être plutôt considéré comme une sorte de charnière) d'un côté, et l'être humain, la vie humaine et la culture littéraire (au centre des livres VI-X et, au moins, d'une partie du livre V) de l'autre. Cette apparente solidité interne et l'ampleur de l'éventail des matières ne doivent pas toutefois nous tromper : le recueil révèle en son intérieur un manque étonnant d'équilibre dans le choix et le traitement des sujets, et un critère d'assemblage des questions qui révèle une certaine hiérarchie interne des arguments.

Essayons maintenant de mieux expliquer ce que je viens d'affirmer. Si on observe la structure de l'ouvrage et la séquence des domaines d'analyse et de recherche, on remarque que le monde végétal et animal ne se voit pas consacrer une section particulière, mais que, par exemple, les questions liées aux animaux et en particulier à l'anatomie animale, sont discutés à l'intérieur du livre V, à savoir dans une partie spécifique qui concerne, dans une vision comparative, les caractéristiques du corps humain et les particularités de la vie corporelle des êtres animés. Le livre V inclut donc, parmi d'autres, un groupe des questions comparant l'homme et l'animal en général, telles que la question 27, "Quale animale sia più simile all'uomo », la 28, «Qual eccettuando l'huomo possa chiamarsi più felice animale », la 29 "Qual sia il più crudo animale », la 30 "Qual sia il più lussurioso animale », la 31 "Perche alcune madri inferociscano dodo [lege: dopo] il parto, e altre nò ${ }^{29}$. Par conséquent, on peut déjà voir que l'apparente ambition encyclopédique de reproduire dans ce recueil un savoir universel se traduit de facto en un système scientifique réduit et en même temps sophistiqué, dans lequel les différents arguments sont structurés en utilisant un système hiérarchique divisé en sujets « principaux » et « secondaires ».

Le fait d'avoir mentionné une séquence de questions nous emmène nécessairement à analyser la nature et la structure des unités formées par les questions et les réponses, leur équilibre interne, et la contribution offerte par ces unités à la représentation unitaire d'un élément du monde et d'un domaine du savoir. Dans un article précédent, j'avais déjà remarqué l'intérêt de Tassoni pour tout ce qui curieux, étrange, paradoxal ${ }^{30}$. En même temps toutefois, j'avais aussi relevé que cette nature curieuse des phénomènes décrits par Tassoni n'impliquait ni une recherche consciente du merveilleux et de l'exotique, mais restait enracinée dans la « quotidien », dans les petites merveilles qui caractérisent la vie de tous les jours, et, fait encore plus important, que les curiosités montrées par notre auteur ne dérivaient pas d'une connaissance directe de la nature, mais de la lecture de ses sources, dont il tira non seulement la manière de répondre aux questions mais aussi le sujet lui-même. Sans renier ces conclusions, je voudrais ici aller au delà d'elles, et analyser non pas le sujet des questions et sa nature, mais le modus operandi de Tassoni et ses résultats.

Pour ce qui concerne la structure des questions et l'équilibre entre question et réponse, on peut tout d'abord remarquer, même dans une lecture superficielle, une forte disproportion entre l'apparente banalité de la question et la longueur et la profondeur de la réponse. En réalité, à bien y regarder, les questions ne sont pas banales du tout, soit parce qu'elles proposent des questions de base, soit parce que le problème cache, derrière son apparente futilité, un débat scientifique de taille et l'influence sur la longue durée d'une source centrale. Rappelons ici deux exemples qui exemplifient les deux cas : la première question du livre I, «Se ci sia l'elemento del fuoco », s'interroge sur l'existence du feu. Sa réponse prend 8 pages, et trace une véritable histoire de la théorie des éléments qui va d'Aristote à Bernardino Telesio, critique la vision aristotélicienne des éléments, et traite de toute une série de questions liées à l'existence du feu, à savoir l'existence d'un feu céleste et terrestre, les différences

\footnotetext{
29 Tassoni 1645, V,27-31, p. 127-131. Traduction française: «Quel est l'animal le plus similaire à l'homme ?», «Quel est l'animal le plus heureux, à l'exception de l'homme ? », «Quel est l'animal le plus cruel ? », «Quel est l'animal le plus luxurieux ? », «Pourquoi certaines mères déviennent plus féroces après l'accouchement, et d'autres pas? ».

30 Ventura 2007, p. 303-306.
} 
entre les deux, le mouvement du feu, son poids et son mouvement, la nature de la chaleur ${ }^{31}$. Ce petit traité de physique (qu'on ne peut pas reproduire ici dans son entièreté) se conclut sans une véritable réponse à la question concernant l'existence du feu, mais reprend, d'un point de vue philosophique et scientifique, tous les éléments du débat scolastique et savant développé à travers les siècles autour de la nature du feu, de sa position dans le système des éléments et dans la philosophie naturelle d'Aristote à Telesio, et de sa division entre feu céleste et terrestre. Par conséquent, on peut dire que, dans les Pensieri diversi, le rapport entre question apparemment banale et réponse fortement érudite et complexe n'est pas simplement disproportionné, ni que la première représente simplement un prétexte au développement érudit de la seconde, mais que Tassoni, fort d'une érudition scientifique profonde, a choisi de manière rationnelle des questions qui touchent au cœur du débat scientifique et savant, et lui permettent d'explorer son histoire et son évolution.

Un exemple du second cas se retrouve dans les parties du texte qu'on serait tenté de définir comme des divertissements érudits à dimension ludique, mais qui sont, en y regardant mieux, reprises telles quelles d'une autre collection de problèmes ou d'un traité de philosophie naturelle. La question VII du livre I, "Perche il pane paia più bianco raffreddato, che mentre è caldo », est accompagnée d'une réponse qui débute avec le rappel de la source dont elle est dérivée, les Problemata pseudoaristotéliciens $^{32}$. Dans la réponse, Tassoni reprend le procède logique du Pseudo-Aristote et de Pierre d'Abano, et les discussions concernant l'association problématique des couleurs blanche et noire à la chaleur et au froid. En fait, tant la chaleur que le froid peuvent être associés dans le monde de la nature aux deux couleurs, et la difficulté se retrouve dans l'identification correcte du lien «naturel » et « accidentel ». Dans ce second cas, où une question apparemment banale est suivie d'une longue réponse (qu' on ne peut pas reproduire ici dans son entièreté), on voit clairement comment le problème est choisi non seulement parce qu'il touche, dans sa simplicité, une question de fond, mais surtout car il reprend une longue tradition textuelle. Cette dernière est représentée par le Pseudo-Aristote, le commentaire de Pierre d'Abano, et les discussions autour de la question menée par Girolamo Cardano dans le De subtilitate (1550-1554) et par son principal adversaire, Julius Caesar Scaliger dans les Exercitationes exotericae de subtilitate $(1557)^{33}$. Ce second cas montre clairement qu'on ne peut pas parler simplement ni de disproportion ou de déséquilibre entre question et réponse, ni de surcharge érudite de la réponse, mais qu'on doit bien identifier la fonction de cette érudition. Dans le cas de Tassoni, il ne s'agit pas d'une simple décoration, mais un rappel à une tradition philosophique et scientifique précise et, en même temps, une démonstration ultérieure de l'attitude consciente et savante - on pourrait presque dire « livresque »- qui a guidé l'activité de notre compilateur.

Ayant rappelé la présence et la fonction des sources, on peut considérer qu'on a déjà traité du deuxième critère de catalogage des recueils de questions-réponses, l'analyse des sources. Tassoni se sert, il est évident, d'un florilège d'autorités principales, parmi lesquelles on trouve Aristote, Girolamo Cardano (surtout le De subtilitate), Julius Caesar Scaliger (et ses Exercitationes), et Bernardino Telesio. Ce qu'on peut ajouter, ou mieux rappeler, est que ces autorités ne sont pas citées seulement en tant qu'auteurs de textes de philosophie naturelle et références phares de la culture scientifique contemporaine, mais aussi en tant que modèles de processus logique, de raisonnement. C'est ce même raisonnement logique que Tassoni s'efforce de suivre dans son interprétation des phénomènes naturels tels qu'ils sont représentés dans les livres qu'il consulte. Par conséquent, dans le cas de Tassoni, la présence des sources ne doit pas être vue en termes d'opposition à la logique et à la rationalité. Au

\footnotetext{
31 Tassoni 1645, I,1, p. 1-8.

32 Ibid., p. 14-16. Traduction française : « Pourquoi le pain semble être plus blanc quand il est froid que quand il est chaud ? ». La source originaire est identifiable en Ps. Aristoteles, Problemata, XXI,4, Parisiis, 1520, ici f. 142vb-143ra (avec le commentaire intitulé Expositio succincta Problematum écrit par Pierre d'Abano).

33 Sur le De subtilitate de Cardano, voir les monographies de Schütze 1999 et Grafton 1999.
} 
contraire, les textes de références offrent non seulement du matériel et des informations fiables et certifiées par leur autorité, mais aussi un modèle de raisonnement à suivre.

En conclusion, l'exemple des Pensieri diversi de Tassoni a montré en premier lieu que notre impression d'avoir affaire avec un recueil de questions-réponses diamétralement différent des Problemas de Lopez de Villalobos peut être confirmée. Mais il a d'autre part aussi montré qu'on ne peut pas réduire cette différence à une simple opposition entre didactique et discours savant, entre raisonnement/rationalité et érudition/discours savant. Au contraire, on ne peut que remarquer que ces éléments se croisent et se mêlent, et que procédé logique et culture philosophique et scientifique trouvent leur point de rencontre dans les recueils de questions-réponses, et non seulement pour offrir à leurs lecteurs une didactique simple et accessible, mais aussi une formation à l'approche rationnelle du monde de la nature et des sciences.

\section{Conclusions}

En conclusion, nous avons montré que les critères choisis, quoique partiels et nécessitant d'autres éléments d'analyse, peuvent contribuer à notre entreprise de dresser un catalogue de ces textes, de caractériser cette typologie de texte dans son ensemble et de distinguer des sous-typologies, et enfin de fournir un système d'orientation et de navigation à l'intérieur d'un genre littéraire probablement très vaste et varié. Les trois critères servent en outre à éclaircir la contribution de ces textes, en tant que forme d'encyclopédisme et de structure textuelle parallèle aux textes encyclopédiques, à la transmission et à la diffusion de la culture scientifique. Cette culture scientifique, ils ne la transmettent pas seulement de façon «non ambiguë et autoritaire », mais aussi d'une façon «problématisante », ou mieux, en tant que forme d'exercice à la pratique de l'analyse et de l'interprétation rationnelle de la réalité.

\section{Références}

Arrizabalaga, J. Francisco Lopez de Villalobos (c. 1473 - c. 1549, medico cortesano, "Dynamis. Acta Hispanica ad Medicinae Scientiarumque Historiam Illustrandam”, 22 (2002), p. 29-58.

Bergdolt, K. et Pfister, M. (2011). Dialoge zwischen Wissenschaft, Kunst und Literatur in der Renaissance, ed. K: Bergdolt et M. Pfister, Wiesbaden: Allemagne, Harrassowitz, 2011 (Wolfenbütteler Abhandlungen zur Renaissanceforschung, 27).

Blair, A. (1999a). Authorship in the Popular "Problemata Aristotelis ", "Early Science and Medicine", 4 (1999), p. 190-227.

Blair, A. (1999b). The Problemata as a Natural Philosophical Genre, in Natural Particulars. Nature and Disciplines in Renaissance Europe, ed. A. Grafton et N. C. Siraisi, Cambridge-London: Etats-UnisAngleterre, MIT Press, 1999 (Dibner Institute Studies in the History of Science and Technology, 1), p. 171-204.

Cardelle de Hartmann, C. (2007). Lateinische Dialoge 1200-1400: literaturhistorische Studie und Repertorium, Leiden - Boston: Pays-Bas-Etats Unis, Brill, 2007 (Mittellateinische Studien und Texte, 37).

Conrad d'Halberstadt (1476). Responsorium curiosorum, Lübeck: Allemagne, Brandis, 1476.

De Callataÿ, G. et Van den Abeele, B. (2009). Une lumière venue d'ailleurs. Héritages et ouvertures dans les encyclopédies d'Orient et d'Occident au Moyen Age. Actes du colloque de Louvain-laNeuve, 19-21 mai 2005, éd. G. De Callataÿ et B. Van den Abeele, Turnhout:Belgique, Brepols, 2009 (Réminisciences, 9).

Fassò, L. (1937). Alessandro Tassoni, « Enciclopedia italiana» en ligne (version imprimée publiée en 1937): http://www.treccani.it/enciclopedia/alessandro-tassoni_\%28EnciclopediaItaliana\%29/ 
Grafton, A. (1999). Cardano's Cosmos. The Worlds and Works of a Renaissance Astrologer. Harvard: Etats Unis, Harvard University Press, 1999.

Hempfer, K.W. et al. (2002). Möglichkeiten des Dialogs: Struktur und Funktion einer literarischen Gattung zwischen Mittelalter und Renaissance in Italien, éd. K. W. Hempfer et al., Stuttgart: Allemagne, Steiner Verlag, 2002 (Text und Kontext, 15).

Horst, G. (1621). Conciliator enucleatus seu differentiarum philosophicarum et medicarum, Gießen: Allemagne, Chemlinus, 1621.

Jacobi, I. (1662). Problemata miscellanea circa Fundamenta Medicinae Physiologicae Occurrentia, Jena : Allemagne, Neunhahnius, 1662.

Jannaco, C. (1966). Studi tassoniani : atti e memorie del Convegno Nazionale di Studi per il IV Centenario della Nascita di Alessandro Tassoni (Modena, 6-7 novembre 1965), ed. C. Jannaco, Modena: Italia, Aedes Muratoriana, 1966 (Biblioteca della Deputazione di Storia Patria per le Antiche Province Modenesi, N.S., 6).

Klotz, F. et Oikonomopoulou, K. (2011). The Philosopher's banquet: Plutarch's Table Talk in the Intellectual Culture of the Roman Enmpire, ed. F. Klotz et K. Oikonomopoulou, Oxford et al.: Royaume-Uni et al., Oxford University Press, 2011.

König, J. et Woolf, G. (2013). Encyclopaedism from Antiquity to the Renaissance, ed. J. König et G. Woolf, Cambridge: Royaume Uni, Cambridge University Press, 2013.

Lawn, B. (1963). The Salernitan Questions. An introduction to the History of Medieval and Renaissance Problem literature, Oxford: Royaume Uni, Oxford University Press, 1963.

Lopez de Villalobos, F. (1543). Libro intitulado Problemas que tracta de cuerpos naturales y morales, Sevilla: Espagne, 1543.

Meier, C. (2002). Die Enzyklopädie im Wandel vom Hochmittelalter bis zur frühen Neuzeit. Akten des Kolloquiums des Projekts D im Sonderforschungsbereich 231 (29.11.-1.12. 1996), ed. C. Meier, München: Allemagne, Wilhelm Fink Verlag, 2002 (Münstersche Mittelalter-Schriften, 78).

Picone, B. (1994). L'enciclopedismo medievale. Atti del Colloquio San Gimignano 8-10 Ottobre 1992, ed. M. Picone, Ravenna: Italia, Longo Editore, 1994 (Memoria del tempo, 1).

Schütze, I. (1999). Die Naturphilosophie in Girolamo Cardano's "De subtilitate". München: Allemagne, Wilhelm Fink Verlag, 1999 (Humanistische Bibliothek. Reihe I: Abhandlungen, 49).

Pseudo-Aristote, Problemata, Parisiis, 1520 (=Pseudo-Aristotelis Problemata cum dpulici [!] translatione, Parisiis, 1520).

Tassoni, A. (1645). De' Pensieri diversi di Alessandro Tassoni Libri dieci. Corretti, ampliati, e arrichiti per tutto dall'autore di nuove curiosità, Venetia, 1645.

Traninger, A. (2012). Disputation, Deklamation, Dialog: Medien und Gattungen europäischer Wissensverhandlungen zwischen Scholastik und Humanismus, Stuttgart: Allemagne, Steiner Verlag, 2012 (Text und Kontext: romanische Literaturen und allgemeine Literaturwissenschaft, 33).

Ventura, I. (2015). Extraire, organiser, transmettre le savoir dans les encyclopédies du Moyen Âge Tardif: Albert le Grand dans le Speculum naturale de Vincent de Beauvais et la Catena aurea entiumd'Henry d'Herford, dans Lire en extraits. Lecture et production des textes, de l'Antiquité au Moyen Âge, éd. S. Morlet, Paris : France, PUPS, 2015, p. 443-464.

Ventura, I. (2009). On Philosophical Encyclopaedism in the Fourteenth Century: The Catena aurea entium of Henry of Herford, dans Une lumière venue d'ailleurs. Héritages et ouvertures dans les encyclopédies d'Orient et d'Occident au Moyen Age. Actes du colloque de Louvain-la-Neuve, 19-21 mai 2005, ed. G. De Callataÿ et B. Van den Abeele, Turnhout : Belgique, Brepols, 2009 (Réminisciences, 9), p. 199-245.

Ventura, I. (2007). Per modum quaestionis compilatum... The Collections of Natural Questions and their Development from the Thirteenth to the Sixteenth Century, dans All you need to know: Encyclopaedias and the idea of general knowledge (Conference, Prangins, Switzerland, 18-20 Sept. 2003), ed. P. Michel, Aachen: Allemagne, Shaker Verlag, 2007. (En ligne sur le site www . enzyklopaedie.ch) 


\section{SHS Web of Conferences}

Ventura, I. (2004). Quaestiones and Encyclopedias. Some aspects of the Late Medieval Reception of pseudo-Aristotelian Problemata in Encyclopaedic and Scientific Culture, dans Schooling and Society. The Ordering and Reordering of Knowledge in the Western Middle Ages, ed. A.A. MacDonald M.W. Twomey, Leuven-Paris-Dudley: Belgique et al., Peeters, 2004 (Groningen Studies in Cultural Change, 6), p. 23-42.

Zucker, A. (2014). Encyclopédire. Formes de l'ambition encyclopédique dans l'Antiquité et au Moyen Âge, ed. A. Zucker, Turnhout: Belgique, Brepols, 2014 (Collection d'Études médiévales de Nice, 14). 